\title{
Drought Resistance and Mitotic Instability of Tritipyrum Compared with Triticale and Bread Wheat
}

\author{
Zolfaghar SHAHRIARI'*, Mohammad Taghi ASSAD ${ }^{1}$, Hosein Shahsavand HASANI² \\ ${ }^{1}$ Shiraz University, Department of Agronomy and Plant Breeding, College of Agriculture, \\ Shiraz,Iran;Shahriari225@gmail.com (*correspondingauthor) \\ ${ }^{2}$ Shahid Bahonar University, Department of Agronomy and Plant Breeding, College of Agriculture, Kerman, Iran
}

\begin{abstract}
This study presents the first data on the drought resistance pattern of seven new synthetic 6x primary Tritipyrum amphiploid lines and evaluates their mitotic instability. The primary Tritipyrum lines were crossed with Iranian 6x bread wheat 'Navid' cultivar and their $\mathrm{F}_{1}$ and $\mathrm{F}_{2}$ progenies were obtained. Two experiments with complete randomized design were conducted under optimum and limited water conditions to evaluate Tritipyrum-derived genotypes for drought resistance in greenhouse. Under optimum water conditions, grain yield, numbers of grains per spike and harvest index of Tritipyrum-derived genotypes were significantly lower than bread wheat; however the differences were not significant under limited water conditions. These results showed the better responses of Tritipyrumderived genotypes to drought conditions. Evaluation of leaf osmotic and water potentials and drought susceptibility index showed that drought resistance of Tritipyrum and $\mathrm{F}_{1}$ genotypes was significantly higher than that of bread wheat and Triticale. Cytological investigations showed that Tritipyrum-derived genotypes aneuploidy was significantly higher than Triticale and bread wheat $(p<0.05)$. Mitotic instability in light grains (1000-grains weight $<30 \mathrm{gr}$ ) was significantly higher than heavy grains (1000-grains weight $>30 \mathrm{gr})$ in parental and $\mathrm{F}_{2}$ genotypes $(p<0.01)$. Aneuploidy has showed a significant negative correlation with fertility, grain yield and 1000-grains weight in Tritipyrum genotypes. In general, Tritipyrum may complement the role of bread wheat in arid and semi-arid regions; but further breeding research is needed to overcome its mitotic instability.
\end{abstract}

Keywords: amphiploid, aneuploidy, drought susceptibility index

\section{Introduction}

Adverse environmental conditions and abiotic stresses are major limiting factors for wheat production in arid and semi-arid areas (Talebi et al., 2009). Synthesis of $6 \mathrm{x}$ amphiploid cereals with higher tolerance against abiotic stresses is needed to supply the increasing world population with the required amount of wheat production (Kamyab et al., 2009).

New cereal, primary Tritipyrum lines $(2 \mathrm{n}=6 \mathrm{x}=42$, $\left.\mathrm{AABBE}^{\mathrm{b}} \mathrm{E}^{\mathrm{b}}\right)$ is a synthetic amphiploid between Triticum durum (AABB) and Thinopyrum bessarabicum species $\left(\mathrm{E}^{\mathrm{b}} \mathrm{E}^{\mathrm{b}}\right)$ in which the introduction of alien genetic material from Thinopyrum genus into wheat through wide crosses has provided increased genetic diversity and thereby an opportunity to improve traits such as drought and salinity tolerance and disease resistance (Allahdoo et al., 2009; Chen Q, 2005; Shahsavand Hassani et al., 2000).

Primary Tritipyrum lines, which was produced at John Innes Centre, Norwich, UK (Kamyab et al., 2009; King et al., 1997) is the third man made and salt tolerant synthetic crop after Triticale and Tritordeum, to be evaluated for conditions unsuitable for wheat growth, particularly, at the flowering stage (Shahsavand Hassani et al., 2000). The genetic diversity and adaptation of wheat and Triticale have been studied quite extensively, but such assessments are still far from enough in the case of primary Tritipyrum lines. Improved grain yield is a critical criterion which can be determined by measuring yield components and other closely associated characteristics (Kamyab et al., 2009; Sharma et al., 2003).

Having disadvantages such as mitotic instability, primary Tritipyrum lines still may not be considered as a commercial salt tolerant crop. Primary Tritipyrum lines tends to suffer problems similar to those of Triticale in its early days, such as chromosome instability and low fertility, which in Triticale were overcome by means of breeding practices (Shahsavand Hassani et al., 1998; Shahsavand Hassani et al., 2000).

The assessment of today's Tritipyrum situation in comparison with Triticale and the possible problems facing breeders in future is not an easy undertaking. The comprehensive and voluminous work performed on Triticale is manifested interview papers on different aspects of that plant, and shows the importance of this first man-made cereal. With due research, the primary Tritipyrum lines have the same potential to become a success story of another man made cereal (Shahsavand Hassani et al., 2000).

The aim of this study was to determine the drought resistance and mitotic instability of a number of primary Tritipyrum lines and to evaluate bread wheat/ Tritipyrum progenies potential for drought resistance breeding in 
bread wheat. This study presents the first data on the drought resistance pattern of primary 6x Tritipyrum amphiploid lines.

\section{Materials and methods}

The experiment was carried out in the green house and cytogenetic laboratory of department of agronomy and plant breeding, Shiraz University, Shiraz, Iran.

\section{Greenhouse trials}

Primary Tritipyrum lines $[\mathrm{Az} / \mathrm{b} ; \mathrm{La} / \mathrm{b} ;(\mathrm{Ka} / \mathrm{b})(\mathrm{Cr} / \mathrm{b})$, $\left.\left.\mathrm{F}_{5} ;(\mathrm{Ma} / \mathrm{b})(\mathrm{Cr} / \mathrm{b}) ;(\mathrm{St} / \mathrm{b})(\mathrm{Cr} / \mathrm{b}), \mathrm{F}_{3} ; \mathrm{Ma} / \mathrm{b}, \mathrm{Cr} / \mathrm{b}\right)\right]$ were crossed with Iranian bread wheat 'Navid' cultivar. The seeds of primary lines were selected based on previous studies in Iran (Kamyab et al., 2009; Shahsavand Hassani et al., 2000). Spikes of seven primary Tritipyrum lines $\left(2 n=6 x=42, A_{A B B E}^{b} E^{b}\right)$ were emasculated and then pollinated with pollen from Iranian bread wheat 'Navid' cultivar, and $F_{1}$ seeds of each cross were produced. The seven primary Tritipyrum lines, their $\mathrm{F}_{1}$ progenies and two controls including promising Triticale 4118 line and Iranian bread wheat 'Navid' cultivar were sown in greenhouse at $5 \mathrm{~kg}$ pots in two experiments with completely randomized design with 3 replications and 3 samples in each experimental unit. The soil was loamy clay with a field capacity of $27 \%$. The two experiments differed with respect to their irrigation regimes. Pots were irrigated twice a week to $20 \%$ (stress experiment) and 60\% (non-stress experiment) of $100 \%$ soil water capacity, respectively. Grain yield per plant, 1000 grains weight (gr), biological yield (gr/plant), harvest index and fertility were measured. Fertility was expressed as the number of grains per spikelet measured on the main spike of plant with longest shoot. For drought resistance investigation, leaf water potential and osmotic potential were measured and drought susceptibility index was determined. Leaf water potential $\left(\psi_{\mathrm{w}}\right)$ was measured using a PMS pressure bomb (PMS Instrument Co., Corvallis, OR) at stem elongation, booting and flowering stages based on Zadoks's Code (Zadoks et al., 1974 ) in both experiments. The youngest fully expanded leaf was detached and placed rapidly in a sample chamber and the pressure was recorded. Three randomly selected plants were used for each developmental stage. Measurements were completed between 13:00 and 15:00 o'clock. To measure osmotic potential $(\psi)$, the youngest fully expanded leaf was used for each developmental stage. Leaves were placed in plastic bags and rapidly packed in a box in order to avoid water loss as evaporation from the sample, and were maintained at $-15^{\circ} \mathrm{C}$ for five hours. The frozen samples were then thawed for approximately 30 minutes and the freezing point $(T)$ of collected saps was measured using a digital thermometer (Model ET-2001). The osmotic potential was then calculated (Kramer, 1995) as $\psi$. $=(-\mathrm{T} / 1.86) \times 2.27)$.

The drought susceptibility index $(S)$ was determined by $S=\left[1-\left(\mathrm{y}_{\mathrm{D}} / \mathrm{y}_{\mathrm{P}}\right)\right] / \mathrm{D}$ equation (Fischer and Mourer,
1978), where $y_{D}$ and $y_{p}$ are the grain yields of each cultivar at stress and non-stress conditions, respectively; and $\mathrm{D}=$ 1- $\left(Y_{D} / Y_{p}\right) . Y_{D}$ and $Y_{P}$ are average yields of all cultivars under stress and non-stress conditions, respectively.

\section{Cytological investigations}

For mitotic chromosome counting study, the primary Tritipyrum lines and their $\mathrm{F}_{2}$ selfing progenies were evaluated in a complete randomized design. The Tritipyrum lines and promising Triticale line were divided into two classes based on their 1000-grains weight (less and higher than $30 \mathrm{gr}$ ). 'Navid' bread wheat cultivar was only classified into the heavy grain class (heavier than $30 \mathrm{gr}$ ). Grains were germinated in Petri dish and then planted in small pots and allowed to grow to a proper size for chromosome counting. The Chromosome number of all lines was counted in 5 metaphase plates by minor modification of the conventional root tip squash protocol (Oudjehih and Boukaboub, 2000; Oudjehih and Bentouati 2006; Tosun, 1999).

\section{Statistical analysis}

All data were subjected to analysis of variance using SPSS statistical software. Means were separated and compared using Duncan multiple range test (DMRT) at the 0.05 level of significance. Mean differences between light and heavy grains euploids were compared using least significant difference (LSD). Pearson correlation coefficient was calculated between variables (Assad, 1997).

\section{Results and discussion}

\section{Drought resistance}

The relative yield performance of all primary Tritipyrum, promising Triticale and bread wheat lines under drought stress and normal conditions is a common starting point in the selection of genotypes for use in breeding in dry environments (Byrne et al., 1995; Rajaram and van Ginkel, 2001). Tritipyrum-derived genotypes and bread wheat did not differ significantly in respect to harvest index $(p>0.05)$, grain yield $(p>0.05)$ and biological yield $(p>0.05)$ under stress condition; however the differences were significant under non-stress conditions (Tab. 1 and 2). Tritipyrum-derived genotypes and Triticale did not differ significantly in respect to harvest index under stress conditions $(p>0.05)$. Biological yield and grain yield of some Tritipyrum-derived genotypes were significantly lower than Triticale under stress and non-stress conditions $(p<0.05)$. Under a particular pressure of environmental stress, cultivars with high yield potential produce less then certain cultivars that have lower yield potential but seem to be better adapted to stress (Blum, 2005). 1000 grains weight of Tritipyrum-derived genotypes was significantly lower than bread wheat and Triticale under stress and nonstress conditions $(p<0.05)$. Number of grains per spike of Tritipyrum-derived genotypes was significantly lower than bread wheat and Triticale under non-stress conditions 
172

Tab. 1. Yield components of 6x Tritipyrum-derived genotypes compared with promising Triticale (4118) and bread wheat 'Navid' cultivar under normal irrigation conditions

\begin{tabular}{|c|c|c|c|c|c|c|c|}
\hline 6x amphiploides & Line/cultivar/progenies & $\begin{array}{l}\text { Grain yield } \\
\text { (gr/ plant) }\end{array}$ & $\begin{array}{c}\text { Fertility } \\
\text { (grain number } \\
\text { / spikelet) }\end{array}$ & $\begin{array}{c}\text { 1000-grains } \\
\text { weight } \\
\text { (gr) }\end{array}$ & $\begin{array}{c}\text { Grain } \\
\text { no/spike }\end{array}$ & $\begin{array}{c}\text { Biological } \\
\text { yield } \\
\text { (gr/plant) }\end{array}$ & $\begin{array}{c}\text { Harvest } \\
\text { index }\end{array}$ \\
\hline \multirow{7}{*}{ 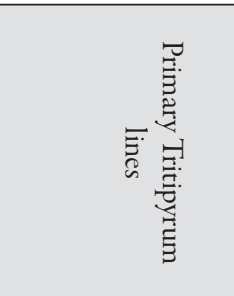 } & $(\mathrm{Ka} / \mathrm{b})(\mathrm{Cr} / \mathrm{b}), \mathrm{F}_{5}$ & $2.93 \mathrm{c}$ & $2.86 \mathrm{~d}$ & $32.87 \mathrm{~d}$ & $35.77 \mathrm{cde}$ & $11.54 \mathrm{cde}$ & $25.43 \mathrm{e}$ \\
\hline & $\mathrm{La} / \mathrm{b}$ & $3.07 \mathrm{bc}$ & $3.07 \mathrm{bcd}$ & $33.7 \mathrm{~cd}$ & $34.56 f$ & $11.43 \mathrm{def}$ & 26.81cde \\
\hline & $\mathrm{Az} / \mathrm{b}$ & $3.03 b c$ & $2.9 \mathrm{dc}$ & $33.74 \mathrm{~cd}$ & $35.07 \mathrm{def}$ & $11.44 \mathrm{def}$ & $26.52 \mathrm{cde}$ \\
\hline & $(\mathrm{Ma} / \mathrm{b})(\mathrm{Cr} / \mathrm{b})$ & $3.13 b c$ & $3.03 \mathrm{bcd}$ & $34.33 \mathrm{~cd}$ & $34.93 \mathrm{ef}$ & $11.93 \mathrm{~b}$ & 26.26de \\
\hline & $(\mathrm{St} / \mathrm{b})(\mathrm{Cr} / \mathrm{b}), \mathrm{F}_{3}$ & $2.98 \mathrm{c}$ & $3.5 \mathrm{a}$ & $33.9 \mathrm{~cd}$ & $35.5 \mathrm{cdef}$ & $11.76 \mathrm{bcd}$ & $25.49 \mathrm{e}$ \\
\hline & $\mathrm{Ma} / \mathrm{b}$ & $2.56 \mathrm{~d}$ & $3.07 \mathrm{bcd}$ & $33.1 \mathrm{~d}$ & $36.17 \mathrm{bc}$ & $11.63 \mathrm{bcde}$ & $22 f$ \\
\hline & $\mathrm{Cr} / \mathrm{b}$ & $2.97 \mathrm{c}$ & $3.26 a b c$ & $34.74 \mathrm{~cd}$ & $35.97 \mathrm{bcd}$ & $11.4 \mathrm{def}$ & 26.31de \\
\hline Triticale & 4118 & $4.03 a$ & $3.4 \mathrm{ab}$ & $45 a$ & $36.9 \mathrm{a}$ & $12.47 \mathrm{a}$ & $32.35 \mathrm{ab}$ \\
\hline Bread wheat & 'Navid' & $4.23 a$ & $3.6 \mathrm{a}$ & $41.5 b$ & $37.33 a$ & $12.8 \mathrm{a}$ & $33.08 \mathrm{a}$ \\
\hline \multirow{9}{*}{ 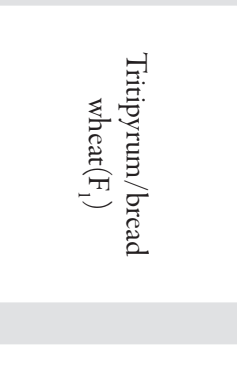 } & $(\mathrm{Ka} / \mathrm{b})(\mathrm{Cr} / \mathrm{b}), \mathrm{F}_{5} / \operatorname{Navid}\left(\mathrm{F}_{1}\right)$ & $3.27 b c$ & 2.1e & $34.7 \mathrm{~cd}$ & $33.01 \mathrm{~g}$ & $11.24 \mathrm{ef}$ & $29.06 \mathrm{~cd}$ \\
\hline & $(\mathrm{La} / \mathrm{b}) / \operatorname{Navid}\left(\mathrm{F}_{1}\right)$ & $3.4 b$ & $1.83 \mathrm{ef}$ & $34.71 \mathrm{~cd}$ & $32.09 \mathrm{~g}$ & $11.46 \mathrm{def}$ & $29.65 b c$ \\
\hline & $(\mathrm{Az} / \mathrm{b}) / \operatorname{Navid}\left(\mathrm{F}_{1}\right)$ & $3.1 \mathrm{bc}$ & $2.07 \mathrm{ef}$ & $33.6 \mathrm{~cd}$ & $32.5 \mathrm{~g}$ & $11.12 \mathrm{f}$ & $27.9 \mathrm{cde}$ \\
\hline & $(\mathrm{Ma} / \mathrm{b})(\mathrm{Cr} / \mathrm{b}) / \operatorname{Navid}\left(\mathrm{F}_{1}\right)$ & $3.23 \mathrm{bc}$ & $2 \mathrm{ef}$ & $33.97 \mathrm{~cd}$ & $32 \mathrm{~g}$ & $11.5 \mathrm{def}$ & $28.12 \mathrm{cde}$ \\
\hline & $(\mathrm{St} / \mathrm{b})(\mathrm{Cr} / \mathrm{b}), \mathrm{F}_{3} / \operatorname{Navid}\left(\mathrm{F}_{1}\right)$ & $3.26 \mathrm{bc}$ & $1.73 \mathrm{f}$ & $33.57 \mathrm{~cd}$ & $31.01 \mathrm{~h}$ & $11.9 \mathrm{bc}$ & $27.45 \mathrm{cde}$ \\
\hline & $(\mathrm{Ma} / \mathrm{b}) / \operatorname{Navid}\left(\mathrm{F}_{1}\right)$ & $3.03 \mathrm{bc}$ & $2.04 \mathrm{ef}$ & $35.26 \mathrm{c}$ & $31.9 \mathrm{gh}$ & $11.43 \mathrm{def}$ & $26.53 \mathrm{cde}$ \\
\hline & $(\mathrm{Cr} / \mathrm{b}) / \operatorname{Navid}\left(\mathrm{F}_{1}\right)$ & $3.25 b c$ & $1.97 \mathrm{ef}$ & $35.23 \mathrm{c}$ & $32.3 \mathrm{~g}$ & 11.6bcde & $28.16 \mathrm{cde}$ \\
\hline & F test & $2.6^{*}$ & $2.45^{*}$ & $2.53^{*}$ & $4.5^{* *}$ & $4.64^{* *}$ & $5.1^{* *}$ \\
\hline & $\mathrm{CV} \%$ & 8.7 & 9.02 & 10.23 & 8.89 & 7.987 & 8.54 \\
\hline
\end{tabular}

columns with the same letter(s) were not significantly different according to Duncan test at $\mathrm{p} \leq 0.05 .{ }^{*}$ and ${ }^{* *}$ significant at 0.05 and 0.01 , respectively

Tab. 2. Yield components and drought susceptibility index of 6x Tritipyrum-derived genotypes compared with promising Triticale (4118) and bread wheat 'Navid' cultivar under limited water conditions

\begin{tabular}{|c|c|c|c|c|c|c|c|c|}
\hline $\begin{array}{c}6 \mathrm{x} \\
\text { amphiploides }\end{array}$ & $\begin{array}{c}\text { Line/cultivar/ } \\
\text { progenies }\end{array}$ & $\begin{array}{l}\text { Grain yield } \\
\text { (gr/ plant) }\end{array}$ & $\begin{array}{l}\text { Fertility } \\
\text { (grain number } \\
\text { / spikelet) }\end{array}$ & $\begin{array}{l}\text { 1000-grains } \\
\text { weight } \\
\text { (gr) }\end{array}$ & $\begin{array}{c}\text { Seed } \\
\text { no/spike }\end{array}$ & $\begin{array}{l}\text { Biologic } \\
\text { Yield } \\
\text { (gr/plant) }\end{array}$ & $\begin{array}{l}\text { Harvest } \\
\text { index }\end{array}$ & $\begin{array}{c}\text { Drought } \\
\text { susceptibility } \\
\text { Index }\end{array}$ \\
\hline \multirow{7}{*}{ 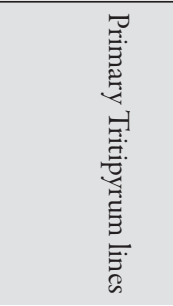 } & $(\mathrm{Ka} / \mathrm{b})(\mathrm{Cr} / \mathrm{b}), \mathrm{F}_{5}$ & $2.07 \mathrm{~b}$ & $2.7 \mathrm{cde}$ & $28.13 \mathrm{de}$ & a33.3 & $8.93 b c$ & $23.13 a$ & $0.425 \mathrm{~cd}$ \\
\hline & $\mathrm{La} / \mathrm{b}$ & $2.1 \mathrm{~b}$ & $2.63 \mathrm{cde}$ & $26.5 \mathrm{e}$ & a33 & $9.03 \mathrm{ab}$ & $23.28 \mathrm{a}$ & $0.455 \mathrm{bcd}$ \\
\hline & $\mathrm{Az} / \mathrm{b}$ & $2.27 \mathrm{ab}$ & $3.16 \mathrm{ab}$ & $27.98 \mathrm{de}$ & a32 & $8.83 b c$ & $25.65 a$ & $0.362 \mathrm{~cd}$ \\
\hline & $(\mathrm{Ma} / \mathrm{b})(\mathrm{Cr} / \mathrm{b})$ & $2.26 \mathrm{ab}$ & $2.6 \mathrm{cde}$ & $28.9 \mathrm{~cd}$ & a32.1 & $8.96 b c$ & $25.26 \mathrm{a}$ & $0.4 \mathrm{~cd}$ \\
\hline & $(\mathrm{St} / \mathrm{b})(\mathrm{Cr} / \mathrm{b}), \mathrm{F}_{3}$ & $2.09 \mathrm{~b}$ & $3.3 a$ & $28.73 \mathrm{~cd}$ & a32.9 & $8.67 \mathrm{c}$ & $24.25 \mathrm{a}$ & $0.431 d$ \\
\hline & $\mathrm{Ma} / \mathrm{b}$ & $2.33 \mathrm{ab}$ & $2.93 \mathrm{bc}$ & $28.78 \mathrm{~cd}$ & a32 & $9.13 \mathrm{ab}$ & $25.54 \mathrm{a}$ & $0.321 \mathrm{~d}$ \\
\hline & $\mathrm{Cr} / \mathrm{b}$ & $2.17 \mathrm{ab}$ & $2.6 \mathrm{cde}$ & $28.6 \mathrm{~cd}$ & a32.5 & $8.9 \mathrm{bc}$ & $24.36 \mathrm{a}$ & $0.402 \mathrm{~cd}$ \\
\hline Triticale & 4118 & $2.4 \mathrm{a}$ & $2.43 \mathrm{e}$ & $38.8 \mathrm{a}$ & a33 & $9.3 \mathrm{a}$ & $25.73 a$ & $0.584 a b$ \\
\hline Bread wheat & 'Navid' & $2.33 \mathrm{ab}$ & $2.8 \mathrm{~cd}$ & $36.03 b$ & a32 & $9.07 \mathrm{ab}$ & $25.74 a$ & $0.649 \mathrm{a}$ \\
\hline \multirow{9}{*}{ 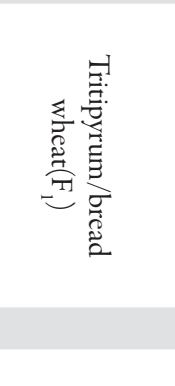 } & $(\mathrm{Ka} / \mathrm{b})(\mathrm{Cr} / \mathrm{b}), \mathrm{F}_{5} / \mathrm{Navid}\left(\mathrm{F}_{1}\right)$ & $2.3 \mathrm{ab}$ & $2.39 \mathrm{e}$ & $30.4 \mathrm{bc}$ & $33.1 \mathrm{a}$ & $8.8 \mathrm{bc}$ & $26.14 a$ & $0.427 \mathrm{bcd}$ \\
\hline & $(\mathrm{La} / \mathrm{b}) / \operatorname{Navid}\left(\mathrm{F}_{1}\right)$ & $2.16 \mathrm{ab}$ & $2.46 \mathrm{e}$ & $30.2 c$ & $32 \mathrm{a}$ & $9.03 \mathrm{ab}$ & $23.98 \mathrm{a}$ & $0.521 \mathrm{abc}$ \\
\hline & $(\mathrm{Az} / \mathrm{b}) / \operatorname{Navid}\left(\mathrm{F}_{1}\right)$ & $2.4 \mathrm{a}$ & $2.6 \mathrm{cde}$ & $30.3 c$ & $31.5 \mathrm{a}$ & $9.06 \mathrm{ab}$ & $26.46 a$ & $0.324 \mathrm{~d}$ \\
\hline & $(\mathrm{Ma} / \mathrm{b})(\mathrm{Cr} / \mathrm{b}) / \operatorname{Navid}\left(\mathrm{F}_{1}\right)$ & $2.13 \mathrm{ab}$ & $2.63 \mathrm{cde}$ & $30.33 c$ & $32.1 \mathrm{a}$ & $9.04 \mathrm{ab}$ & $23.61 \mathrm{a}$ & $0.491 b c$ \\
\hline & $(\mathrm{St} / \mathrm{b})(\mathrm{Cr} / \mathrm{b}), \mathrm{F}_{3} / \operatorname{Navid}\left(\mathrm{F}_{1}\right)$ & $2.2 \mathrm{ab}$ & $2.38 \mathrm{e}$ & $30.13 c$ & $32.5 \mathrm{a}$ & $8.94 \mathrm{bc}$ & $24.68 \mathrm{a}$ & $0.474 \mathrm{bcd}$ \\
\hline & $(\mathrm{Ma} / \mathrm{b}) / \operatorname{Navid}\left(\mathrm{F}_{1}\right)$ & $2.26 \mathrm{ab}$ & $2.41 \mathrm{e}$ & $29.73 \mathrm{~cd}$ & $33.3 a$ & $8.8 \mathrm{bc}$ & $25.77 \mathrm{a}$ & $0.362 \mathrm{~cd}$ \\
\hline & $(\mathrm{Cr} / \mathrm{b}) / \operatorname{Navid}\left(\mathrm{F}_{1}\right)$ & $2.15 \mathrm{ab}$ & $2.44 \mathrm{e}$ & $30.13 c$ & $33.4 \mathrm{a}$ & $8.92 b c$ & $24.07 \mathrm{a}$ & $0.494 \mathrm{bc}$ \\
\hline & F test & $1.01^{\mathrm{ns}}$ & $2.33^{*}$ & $2.36^{*}$ & $1.32^{\mathrm{ns}}$ & $2.37^{*}$ & $0.99^{\mathrm{ns}}$ & $8.22^{* *}$ \\
\hline & $\mathrm{CV} \%$ & 9.05 & 9.65 & 8.98 & 10.8 & 10.43 & 10.87 & 9.32 \\
\hline
\end{tabular}

columns with the same letter(s) were not significantly different according to Duncan multiple range test at $\mathrm{p} \leq 0.05 .{ }^{*}$ and ${ }^{* *}$ significant at 0.05 and 0.01 , respectively

$(p<0.01)$; however it was similar to that of bread wheat under stress conditions $(p>0.05)$. Fertility of some Tritipyrum-derived genotypes was significantly higher than Triticale and bread wheat under limited water conditions $(p<0.05)$. The results were in agreement with several stud- ies that indicated grains per spike and spikes per square meter were the yield components most sensitive to drought; while grain weight remains relatively stable (Guinta et al., 1993; Zhong and Rajaram, 1994). Bread wheat and Triticale yield components was more sensitive to drought 
Tab. 3. Leaf water potential ( $\psi s)$ of $6 x$ Tritipyrum-derived genotypes compared with promising Triticale (4118) and bread wheat 'Navid' cultivar in different growth stages under non-tress and drought stress conditions

\begin{tabular}{|c|c|c|c|c|c|c|c|}
\hline \multirow{2}{*}{ 6x amphiploides } & \multirow{2}{*}{ Line/cultivar/progenies } & \multicolumn{3}{|c|}{ Non-stress conditions } & \multicolumn{3}{|c|}{ Stress conditions } \\
\hline & & Stem elongation & Booting & Flowering & Stem elongation & Booting & Flowering \\
\hline \multirow{7}{*}{ 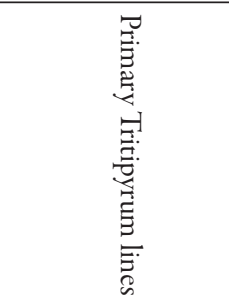 } & $(\mathrm{Ka} / \mathrm{b})(\mathrm{Cr} / \mathrm{b}), \mathrm{F}_{5}$ & $-0.593 \mathrm{~d}$ & $-1.75 \mathrm{~d}$ & $-2.11 \mathrm{~h}$ & $-2.4 \mathrm{~cd}$ & $-3.11 \mathrm{e}$ & $-4.193 \mathrm{~g}$ \\
\hline & $\mathrm{La} / \mathrm{b}$ & $-0.59 \mathrm{~d}$ & $-1.743 d$ & $-2.09 \mathrm{gh}$ & $-2.13 c d$ & $-3.14 \mathrm{e}$ & $-4.2 \mathrm{~g}$ \\
\hline & $\mathrm{Az} / \mathrm{b}$ & $-0.598 \mathrm{~d}$ & $-1.737 \mathrm{c}$ & $-2.08 \mathrm{fgh}$ & $-2.1 \mathrm{c}$ & $-3.11 \mathrm{e}$ & $-4.15 f$ \\
\hline & $(\mathrm{Ma} / \mathrm{b})(\mathrm{Cr} / \mathrm{b})$ & $-0.591 \mathrm{~d}$ & $-1.727 d$ & $-2.07 \mathrm{fgh}$ & $-2.33 d$ & $-3.1 \mathrm{e}$ & $-4.18 f g$ \\
\hline & $(\mathrm{St} / \mathrm{b})(\mathrm{Cr} / \mathrm{b}), \mathrm{F}_{3}$ & $-0.61 d$ & $-1.76 \mathrm{~d}$ & $-2.04 f$ & $-2.12 \mathrm{~cd}$ & $-3.13 e$ & $-4.176 f g$ \\
\hline & $\mathrm{Ma} / \mathrm{b}$ & $-0.603 \mathrm{~d}$ & $-1.747 \mathrm{~d}$ & $-2.057 \mathrm{fg}$ & $-2.15 \mathrm{~cd}$ & $-3.12 \mathrm{e}$ & $-4.21 \mathrm{~g}$ \\
\hline & $\mathrm{Cr} / \mathrm{b}$ & $-0.601 d$ & $-1.74 \mathrm{~d}$ & $-2.05 f$ & $-2.11 \mathrm{c}$ & $-3.1 \mathrm{e}$ & $-4.18 f g$ \\
\hline Triticale & 4118 & $-0.43 b$ & $-1.26 b$ & $-1.42 b$ & $-1.42 \mathrm{a}$ & $-2.82 b$ & $-3.87 b$ \\
\hline Bread wheat & 'Navid' & $-0.337 \mathrm{a}$ & $-1.07 \mathrm{a}$ & $-1.24 a$ & $-1.32 \mathrm{a}$ & $-2.46 \mathrm{a}$ & $-3.62 \mathrm{a}$ \\
\hline \multirow{9}{*}{ 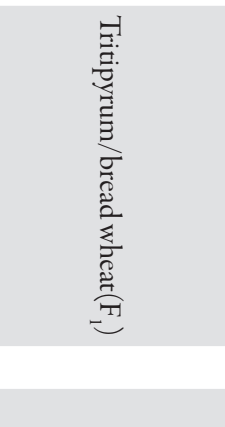 } & $(\mathrm{Ka} / \mathrm{b})(\mathrm{Cr} / \mathrm{b}), \mathrm{F}_{5} / \operatorname{Navid}\left(\mathrm{F}_{1}\right)$ & $-0.49 \mathrm{c}$ & $-1.513 c$ & $-1.617 \mathrm{c}$ & $-1.67 b$ & $-2.93 \mathrm{~d}$ & $-3.65 \mathrm{de}$ \\
\hline & $(\mathrm{La} / \mathrm{b}) / \operatorname{Navid}\left(\mathrm{F}_{1}\right)$ & $-0.48 c$ & $-1.53 c$ & $-1.647 \mathrm{~cd}$ & $-1.73 b$ & $-2.94 \mathrm{~d}$ & $-3.92 \mathrm{~cd}$ \\
\hline & $(\mathrm{Az} / \mathrm{b}) / \operatorname{Navid}\left(\mathrm{F}_{1}\right)$ & $-0.46 b c$ & $-1.53 c$ & $-1.69 \mathrm{e}$ & $-1.83 b$ & $-2.87 \mathrm{c}$ & $-3.95 \mathrm{de}$ \\
\hline & $(\mathrm{Ma} / \mathrm{b})(\mathrm{Cr} / \mathrm{b}) / \operatorname{Navid}\left(\mathrm{F}_{1}\right)$ & $-0.46 b c$ & $-1.523 c$ & $-1.7 \mathrm{e}$ & $-1.8 \mathrm{~b}$ & $-2.9 \mathrm{~cd}$ & $-3.99 \mathrm{e}$ \\
\hline & $(\mathrm{St} / \mathrm{b})(\mathrm{Cr} / \mathrm{b}), \mathrm{F}_{3} / \operatorname{Navid}\left(\mathrm{F}_{1}\right)$ & $-0.487 \mathrm{c}$ & $-1.54 c$ & $-1.673 \mathrm{de}$ & $-1.81 b$ & $-2.89 \mathrm{c}$ & $-3.96 \mathrm{de}$ \\
\hline & $(\mathrm{Ma} / \mathrm{b}) / \operatorname{Navid}\left(\mathrm{F}_{1}\right)$ & $-0.47 \mathrm{c}$ & $-1.547 \mathrm{c}$ & $-1.68 \mathrm{de}$ & $-1.79 b$ & $-2.94 \mathrm{~d}$ & $-3.9 b c$ \\
\hline & $(\mathrm{Cr} / \mathrm{b}) / \operatorname{Navid}\left(\mathrm{F}_{1}\right)$ & $-0.473 c$ & $-1.5 c$ & $-1.68 \mathrm{de}$ & $-1.8 b$ & $-2.903 \mathrm{~cd}$ & $-3.92 \mathrm{~cd}$ \\
\hline & F test & $4.2^{* *}$ & $2.42^{*}$ & $6.3^{* *}$ & $5.43^{* *}$ & $4.17^{* *}$ & $7.21^{* *}$ \\
\hline & $\mathrm{CV} \%$ & 9.86 & 8.43 & 9.78 & 9.45 & 10.06 & 8.52 \\
\hline
\end{tabular}

Columns with the same letter(s) were not significantly different according to Duncan test at $\mathrm{p} \leq 0.05 .{ }^{*}$ and ${ }^{* *}$ significant at 0.05 and 0.01 , respectively

Tab. 4. Leaf osmotic potential of 6x Tritipyrum-derived genotypes compared with promising Triticale (4118) and bread wheat 'Navid' cultivar at different developmental stages under non-stress and drought stress conditions

\begin{tabular}{|c|c|c|c|c|c|c|c|}
\hline \multirow{2}{*}{ 6x amphiploides } & \multirow{2}{*}{ Line/cultivar/progenies } & \multicolumn{3}{|c|}{ Non-stress conditions } & \multicolumn{3}{|c|}{ Stress conditions } \\
\hline & & Stem elongation & Booting & Flowering & Stem elongation & Booting & Flowering \\
\hline \multirow{7}{*}{ 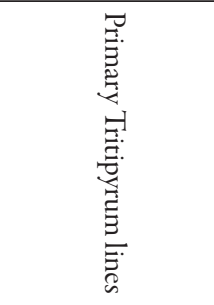 } & $(\mathrm{Ka} / \mathrm{b})(\mathrm{Cr} / \mathrm{b}), \mathrm{F}_{5}$ & $-2.38 f$ & $-3.01 f$ & $-3.65 e$ & $-3.017 d$ & $-3.71 \mathrm{~g}$ & $-4.63 \mathrm{i}$ \\
\hline & $\mathrm{La} / \mathrm{b}$ & $-2.37 f$ & $-2.98 f$ & $-3.62 \mathrm{e}$ & $-2.99 \mathrm{~d}$ & $-3.66 \mathrm{ef}$ & $-4.59 \mathrm{gh}$ \\
\hline & $\mathrm{Az} / \mathrm{b}$ & $-2.39 f$ & $-3.01 f$ & $-3.63 e$ & $-2.97 \mathrm{~d}$ & $-3.68 \mathrm{efg}$ & $-4.6 h i$ \\
\hline & $(\mathrm{Ma} / \mathrm{b})(\mathrm{Cr} / \mathrm{b})$ & $-2.36 f$ & $-2.98 f$ & $-3.61 \mathrm{e}$ & $-2.98 \mathrm{~d}$ & $-3.65 e$ & $-4.58 \mathrm{gh}$ \\
\hline & $(\mathrm{St} / \mathrm{b})(\mathrm{Cr} / \mathrm{b}), \mathrm{F}_{3}$ & $-2.37 f$ & $-2.97 f$ & $-3.6 e$ & $-2.96 \mathrm{~d}$ & $-3.7 \mathrm{fg}$ & $-4.52 \mathrm{f}$ \\
\hline & $\mathrm{Ma} / \mathrm{b}$ & $-2.37 f$ & $-3.02 f$ & $-3.58 \mathrm{e}$ & $-2.95 \mathrm{~d}$ & $-3.68 \mathrm{efg}$ & $-4.56 \mathrm{~g}$ \\
\hline & $\mathrm{Cr} / \mathrm{b}$ & $-2.38 f$ & $-2.99 f$ & $-3.59 \mathrm{e}$ & $-3.02 \mathrm{~d}$ & $-3.69 \mathrm{efg}$ & $-4.57 \mathrm{gh}$ \\
\hline Triticale & 4118 & $-1.46 b$ & $-2.41 b$ & $-2.7 b$ & $-2.51 b$ & $-3.24 b$ & $-4.02 b$ \\
\hline Bread wheat & 'Navid' & $-0.96 \mathrm{a}$ & $-1.91 \mathrm{a}$ & $-2.07 \mathrm{a}$ & $-1.98 \mathrm{a}$ & $-3.79 \mathrm{a}$ & $-3.61 \mathrm{a}$ \\
\hline \multirow{9}{*}{ 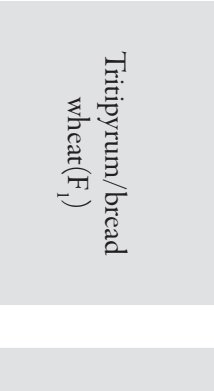 } & $\mathrm{Ka} / \mathrm{b}^{*} \mathrm{Cr} / \mathrm{b}\left(\mathrm{F}_{5}\right)^{*} \operatorname{Navid}\left(\mathrm{F}_{1}\right)$ & $-2.11 \mathrm{de}$ & $-2.6 \mathrm{~d}$ & $-2.94 \mathrm{~cd}$ & $-2.74 c$ & $-3.49 \mathrm{~d}$ & $-4.29 \mathrm{de}$ \\
\hline & $\mathrm{La} / \mathrm{b}^{*} \operatorname{Navid}\left(\mathrm{F}_{1}\right)$ & $-2.09 \mathrm{~cd}$ & $-2.64 \mathrm{de}$ & $-2.98 \mathrm{~d}$ & $-2.73 c$ & $-3.43 c$ & $-4.29 \mathrm{de}$ \\
\hline & $\mathrm{Az} / \mathrm{b}^{*} \operatorname{Navid}\left(\mathrm{F}_{1}\right)$ & $-2.07 \mathrm{c}$ & $-2.66 \mathrm{de}$ & $-2.97 \mathrm{~d}$ & $-2.75 c$ & $-3.46 \mathrm{~cd}$ & $-4.26 \mathrm{cde}$ \\
\hline & $\mathrm{Ma} / \mathrm{b}^{*} \mathrm{Cr} / \mathrm{b}^{*} \operatorname{Navid}\left(\mathrm{F}_{1}\right)$ & $-2.1 \mathrm{cde}$ & $-2.7 \mathrm{e}$ & $-2.93 \mathrm{~cd}$ & $-2.74 \mathrm{c}$ & $-3.44 c$ & $-4.27 \mathrm{cde}$ \\
\hline & $\mathrm{St} / \mathrm{b}^{*} \mathrm{Cr} / \mathrm{b}\left(\mathrm{F}_{3}\right)^{*} \operatorname{Navid}\left(\mathrm{F}_{1}\right)$ & $-2.13 e$ & $-2.51 \mathrm{c}$ & $-2.93 c d$ & $-2.73 c$ & $-3.44 c$ & $-4.257 \mathrm{cde}$ \\
\hline & $\mathrm{Ma} / \mathrm{b}^{*} \operatorname{Navid}\left(\mathrm{F}_{1}\right)$ & $-2.09 \mathrm{~cd}$ & $-2.61 \mathrm{de}$ & $-2.84 \mathrm{c}$ & $-2.69 \mathrm{c}$ & $-3.42 c$ & $-4.253 \mathrm{~cd}$ \\
\hline & $\mathrm{Cr} / \mathrm{b}^{*} \operatorname{Navid}\left(\mathrm{F}_{1}\right)$ & $-2.11 \mathrm{de}$ & $-2.59 \mathrm{~cd}$ & $-2.92 \mathrm{~cd}$ & $-2.71 c$ & $-3.44 c$ & $-4.247 \mathrm{c}$ \\
\hline & F test & $* *$ & $*$ & ** & * & ** & $* *$ \\
\hline & CV\% & 7.94 & 8.71 & 8.93 & 9.01 & 10.31 & 7.87 \\
\hline
\end{tabular}

Columns with the same letter(s) were not significantly different according to Duncan test at $\mathrm{p} \leq 0.05 .{ }^{*}$ and ${ }^{* *}$ significant at 0.05 and 0.01 , respectivel

stress than that of Tritipyrum-derived genotypes. Yield and yield-related traits under stress were independent of yield and yield-related traits under non-stress conditions (Talebi et al., 2009). For most cereals grown under waterlimited conditions the crossover occurs at a yield level of around 2-3t/ha, which is approximately one-third of the yield potential (Blum, 2005).
Tritipyrum-derived genotypes showed lower drought susceptibility indices than bread wheat $(p<0.01)$. Drought susceptibility indices of some Tritipyrum-derived genotypes had no significant difference with Triticale (Tab. 2). Susceptible cultivars on average have higher drought susceptibility indices than intermediate and resistant cultivars (Amiri and Assad, 2005). 
174

Tab. 5. Mean comparison between euploid plants in light grain (1000-grains weight $<30$ gr) and heavy grain (1000-grains weight $>30 \mathrm{gr}$ ) genotypes. Aneuploidy percentage in both grain weight classes is showed as well ( $\mathrm{r}=3$ with 60 seedlings per replication)

\begin{tabular}{|c|c|c|c|c|c|c|}
\hline $\begin{array}{c}6 x \\
\text { amphiploides }\end{array}$ & Line/cultivar/progenies & $\begin{array}{c}\text { Heavy grains } \\
\text { Euploid seedling } \\
\text { number }(2 n=42)\end{array}$ & $\begin{array}{l}\text { Heavy grain } \\
\text { Aneuploidy } \\
(\%)\end{array}$ & $\begin{array}{c}\text { Light grains } \\
\text { Euploid seedling } \\
\text { number }(2 n=42)\end{array}$ & $\begin{array}{c}\text { Light grains } \\
\text { Aneuploidy } \\
\text { (\%) }\end{array}$ & $\begin{array}{l}\text { Mean differences } \\
\text { between light } \\
\text { and heavy grain's } \\
\text { Euploids (LSD) }\end{array}$ \\
\hline \multirow{7}{*}{ 泀 } & $(\mathrm{Ka} / \mathrm{b})(\mathrm{Cr} / \mathrm{b}), \mathrm{F}_{5}$ & $47.3 \mathrm{def}$ & 21.2 & $44.67 \mathrm{bc}$ & 25.6 & 2.63 \\
\hline & $\mathrm{La} / \mathrm{b}$ & 49.5 bcde & 17.5 & $46.3 \mathrm{~b}$ & 22.8 & $3.2^{*}$ \\
\hline & $\mathrm{Az} / \mathrm{b}$ & $51 \mathrm{bcd}$ & 15.0 & $46.7 \mathrm{~b}$ & 22.2 & $4.3^{\prime \prime}$ \\
\hline & $(\mathrm{Ma} / \mathrm{b})(\mathrm{Cr} / \mathrm{b})$ & 49bcde & 18.3 & $45 \mathrm{bc}$ & 25.0 & $4 "$ \\
\hline & $(\mathrm{St} / \mathrm{b})(\mathrm{Cr} / \mathrm{b}), \mathrm{F}_{3}$ & $50 \mathrm{bcde}$ & 16.7 & $46.3 \mathrm{~b}$ & 22.8 & $3.7^{*}$ \\
\hline & $\mathrm{Ma} / \mathrm{b}$ & $51.67 \mathrm{bc}$ & 14.0 & $45.6 \mathrm{~b}$ & 24.0 & $6.07^{\prime \prime}$ \\
\hline & $\mathrm{Cr} / \mathrm{b}$ & $52.7 \mathrm{~b}$ & 12.2 & $46.7 \mathrm{~b}$ & 22.2 & 6 \\
\hline Triticale & 4118 & $58 a$ & 5.0 & $54 \mathrm{a}$ & 5.0 & $4 "$ \\
\hline Bread wheat & 'Navid' & $60 \mathrm{a}$ & 0.0 & - & - & - \\
\hline \multirow{9}{*}{ 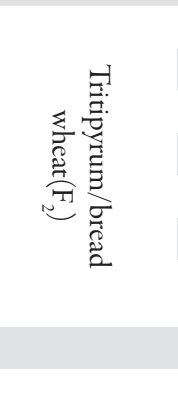 } & $(\mathrm{Ka} / \mathrm{b})(\mathrm{Cr} / \mathrm{b}), \mathrm{F}_{5} / \operatorname{Navid}\left(\mathrm{F}_{2}\right)$ & $45 f$ & 25.0 & $40.7 \mathrm{e}$ & 32.2 & $4.3^{\prime \prime}$ \\
\hline & $(\mathrm{La} / \mathrm{b}) / \operatorname{Navid}\left(\mathrm{F}_{2}\right)$ & $47.3 \mathrm{def}$ & 21.2 & $42.3 \mathrm{cde}$ & 29.5 & 5 \\
\hline & $(\mathrm{Az} / \mathrm{b}) / \operatorname{Navid}\left(\mathrm{F}_{2}\right)$ & $48.3 \mathrm{cdef}$ & 19.5 & $41 \mathrm{de}$ & 31.7 & $7.3^{*}$ \\
\hline & $(\mathrm{Ma} / \mathrm{b})(\mathrm{Cr} / \mathrm{b}) / \operatorname{Navid}\left(\mathrm{F}_{2}\right)$ & $47 \mathrm{ef}$ & 21.7 & $42 \mathrm{cde}$ & 30.0 & 5 \\
\hline & $(\mathrm{St} / \mathrm{b})(\mathrm{Cr} / \mathrm{b}), \mathrm{F}_{3} / \operatorname{Navid}\left(\mathrm{F}_{2}\right)$ & $48.6 \mathrm{cdef}$ & 19.0 & $41 \mathrm{de}$ & 31.7 & $7.6^{\circ}$ \\
\hline & $(\mathrm{Ma} / \mathrm{b}) / \operatorname{Navid}\left(\mathrm{F}_{2}\right)$ & $50.3 \mathrm{bcde}$ & 16.3 & $42.3 \mathrm{cde}$ & 29.5 & $8 *$ \\
\hline & $(\mathrm{Cr} / \mathrm{b}) / \operatorname{Navid}\left(\mathrm{F}_{2}\right)$ & $49.3 \mathrm{bcde}$ & 17.8 & $44 \mathrm{bcd}$ & 26.7 & $5.3^{*}$ \\
\hline & F test & ** & - & ** & - & - \\
\hline & $\mathrm{CV} \%$ & 6.5 & - & 7.1 & - & - \\
\hline
\end{tabular}

Columns with the same letter(s) were not significantly different according to Duncan test at $\mathrm{p} \leq 0.05{ }^{*}$ and ${ }^{* *}$ significant at 0.05 and 0.01 , respectively

Tab. 6. Distribution of Monosomic, nullisomic and Trisomic seedlings among light and heavy grains within the hexaploid Tritipyrum genotypes and their F2 progenies compared with Triticale and bread wheat ( $\mathrm{r}=3$ with 60 seedlings per replication)

\begin{tabular}{|c|c|c|c|c|c|c|c|}
\hline \multirow[b]{2}{*}{ 6x amphiploides } & \multirow[b]{2}{*}{ Line/cultivar/progenies } & \multicolumn{3}{|c|}{ Heavy Grains } & \multicolumn{3}{|c|}{ Light Grains } \\
\hline & & $\begin{array}{c}\text { Monosomic } \\
\text { seedling } \\
(2 \mathrm{n}=41)\end{array}$ & $\begin{array}{l}\text { Trisomic } \\
\text { seedling } \\
(2 \mathrm{n}=43)\end{array}$ & $\begin{array}{c}\text { Nulisomic } \\
\text { seedling } \\
(2 n=40)\end{array}$ & $\begin{array}{c}\text { Monosomic } \\
\text { seedling } \\
(2 \mathrm{n}=41)\end{array}$ & $\begin{array}{l}\text { Trisomic } \\
\text { seedling } \\
(2 \mathrm{n}=43)\end{array}$ & $\begin{array}{c}\text { Nulisomic } \\
\text { seedling } \\
(2 n=40)\end{array}$ \\
\hline \multirow{7}{*}{ 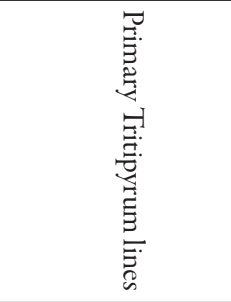 } & $(\mathrm{Ka} / \mathrm{b})(\mathrm{Cr} / \mathrm{b}), \mathrm{F}_{5}$ & $6.3 \mathrm{abc}$ & $1.3 \mathrm{ab}$ & $4.3 \mathrm{abc}$ & $9.3 \mathrm{cde}$ & $1.7 \mathrm{ab}$ & $3.7 \mathrm{bcd}$ \\
\hline & $\mathrm{La} / \mathrm{b}$ & 5bcdef & $1.3 \mathrm{ab}$ & $3.3 \mathrm{bc}$ & $7.3 e$ & $1.3 \mathrm{~b}$ & $3.5 \mathrm{bcd}$ \\
\hline & $\mathrm{Az} / \mathrm{b}$ & $4 \mathrm{defg}$ & $0.7 \mathrm{abc}$ & $3.25 b c$ & $7.27 \mathrm{e}$ & $1 b$ & $3.61 \mathrm{bcd}$ \\
\hline & $(\mathrm{Ma} / \mathrm{b})(\mathrm{Cr} / \mathrm{b})$ & $4.3 \mathrm{cdefg}$ & $1.27 \mathrm{ab}$ & $4 a b c$ & $8.4 \mathrm{de}$ & $1.67 \mathrm{ab}$ & $4 \mathrm{bc}$ \\
\hline & $(\mathrm{St} / \mathrm{b})(\mathrm{Cr} / \mathrm{b}), \mathrm{F}_{3}$ & 4.7bcdefg & $1.32 \mathrm{ab}$ & $3.33 \mathrm{bc}$ & $8.34 \mathrm{de}$ & $1.27 \mathrm{~b}$ & $3 \mathrm{~cd}$ \\
\hline & $\mathrm{Ma} / \mathrm{b}$ & $3.7 \mathrm{efg}$ & labc & $3.27 \mathrm{bc}$ & $8.36 \mathrm{de}$ & $1.01 \mathrm{~b}$ & $3.3 \mathrm{bcd}$ \\
\hline & $\mathrm{Cr} / \mathrm{b}$ & $3 f g$ & $0.68 \mathrm{abc}$ & $3 c$ & 9 cde & $1.32 \mathrm{~b}$ & $2.27 \mathrm{~d}$ \\
\hline Triticale & 4118 & $2.66 \mathrm{~g}$ & $0.33 b c$ & $0 \mathrm{~d}$ & $2.66 \mathrm{f}$ & $0.33 c$ & $0 \mathrm{e}$ \\
\hline Bread wheat & 'Navid' & $0 \mathrm{i}$ & $0 \mathrm{c}$ & $0 \mathrm{~d}$ & - & - & - \\
\hline \multirow{9}{*}{ 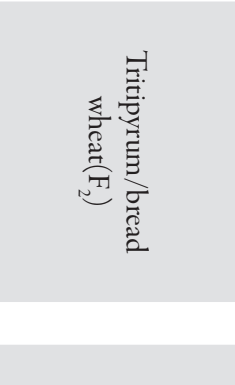 } & $(\mathrm{Ka} / \mathrm{b})(\mathrm{Cr} / \mathrm{b}), \mathrm{F}_{5} / \operatorname{Navid}\left(\mathrm{F}_{2}\right)$ & $7.3 \mathrm{a}$ & $1.35 \mathrm{ab}$ & $5.3 \mathrm{a}$ & $11.7 \mathrm{ab}$ & $1.26 \mathrm{~b}$ & $5.7 \mathrm{a}$ \\
\hline & $(\mathrm{La} / \mathrm{b}) / \operatorname{Navid}\left(\mathrm{F}_{2}\right)$ & 5.3abcde & $1.7 \mathrm{a}$ & $4 a b c$ & $10 \mathrm{bcd}$ & $3 a$ & $3 \mathrm{~cd}$ \\
\hline & $(\mathrm{Az} / \mathrm{b}) / \operatorname{Navid}\left(\mathrm{F}_{2}\right)$ & 6abcd & $0.67 \mathrm{abc}$ & $4.7 \mathrm{abc}$ & $12.4 \mathrm{a}$ & $1.8 \mathrm{ab}$ & $4.59 \mathrm{ab}$ \\
\hline & $(\mathrm{Ma} / \mathrm{b})(\mathrm{Cr} / \mathrm{b}) / \operatorname{Navid}\left(\mathrm{F}_{2}\right)$ & $6.7 \mathrm{ab}$ & $0.33 b c$ & $5 \mathrm{ab}$ & $11 \mathrm{abc}$ & $1.71 \mathrm{ab}$ & $4.3 \mathrm{abc}$ \\
\hline & $(\mathrm{St} / \mathrm{b})(\mathrm{Cr} / \mathrm{b}), \mathrm{F}_{3} / \operatorname{Navid}\left(\mathrm{F}_{2}\right)$ & 5.5abcde & $1 a b c$ & $4 a b c$ & $11.7 \mathrm{ab}$ & $2.3 \mathrm{ab}$ & $4.3 \mathrm{abc}$ \\
\hline & $(\mathrm{Ma} / \mathrm{b}) / \operatorname{Navid}\left(\mathrm{F}_{2}\right)$ & $4.3 \mathrm{cdefg}$ & labc & $3.28 \mathrm{bc}$ & $10 \mathrm{bcd}$ & $2.27 \mathrm{ab}$ & $4.32 \mathrm{abc}$ \\
\hline & $(\mathrm{Cr} / \mathrm{b}) / \operatorname{Navid}\left(\mathrm{F}_{2}\right)$ & 4.7bcdefg & $1.7 \mathrm{a}$ & $4 a b c$ & $10.67 \mathrm{abc}$ & $1 b$ & $3.7 \mathrm{bcd}$ \\
\hline & F test & ** & $*$ & $* *$ & * & $*$ & $*$ \\
\hline & $\mathrm{CV} \%$ & 6.5 & 9.23 & 9.74 & 8.2 & 9.3 & 9.55 \\
\hline
\end{tabular}

Columns with the same letter(s) were not significantly different according to Duncan test at $\mathrm{p} \leq 0.05 .{ }^{*}$ and ${ }^{* *}$ significant at 0.05 and 0.01 , respectively

Tritipyrum parental genotypes showed significantly lower $\psi_{\mathrm{w}}$ values than $\mathrm{F}_{1}$ genotypes, and $\mathrm{F}_{1}$ genotypes showed significantly lower $\psi_{\mathrm{w}}$ values than Triticale and bread wheat at three developmental stages (Tab. 3). Drought resistant genotypes showed lower $\psi_{\mathrm{w}}$ values compared with sensitive genotypes (Amiri and Assad, 2005; 
Tab. 7. Simple correlation coefficients between light and heavy grains aneuploidy, fertility, 1000 grains weight and grain yield of Tritipyrum-derived genotypes

\begin{tabular}{|c|c|c|c|c|}
\hline Traits & $\begin{array}{c}\text { Light grains } \\
\text { aneuploidy (\%) }\end{array}$ & $\begin{array}{c}\text { Heavy grains } \\
\text { aneuploidy (\%) }\end{array}$ & Fertility & $\begin{array}{l}\text { 1000-grains } \\
\text { weight (gr) }\end{array}$ \\
\hline Light grains aneuploidy (\%) & 1 & & & \\
\hline Heavy grains aneuploidy (\%) & $0.935\left(^{* *}\right)$ & 1 & & \\
\hline Fertility & $-0.765\left(^{* *}\right)$ & $-0.681\left(^{* *}\right)$ & 1 & \\
\hline 1000-grains weight (gr) & $\left.-0.879{ }^{(* *}\right)$ & $-0.846\left(^{* *}\right)$ & $0.426\left(^{* *}\right)$ & 1 \\
\hline Grain yield (gr / plant) & $-0.706\left(^{* *}\right)$ & $-0.615\left(^{* *}\right)$ & $0.662\left(^{* *}\right)$ & $\left.0.909{ }^{* *}\right)$ \\
\hline
\end{tabular}

Barlow et al., 1980; Keim and Kronstad, 1981; Moustafa et al., 1996).

The trend of variation in leaf osmotic potential $\left(\psi_{s}\right)$ values in different growth stages for all genotypes was similar to that of $\psi_{\mathrm{w}}$ (Tab. 4). Drought resistant genotypes showed lower $\psi$ values compared with sensitive genotypes (Amiri and Assad, 2005; Blum, 1989; Musick et al., 1994).

\section{Mitotic stability}

Analysis of variance indicated that the effect of grain weight on mitotic chromosome stability was significant. There was a significant difference $(p<0.01)$ between euploid plants means within light and heavy grains of Tritipyrum derived genotypes and Triticale (Tab. 5). The distribution of monosomic, trisomic and nullisomic plants among light- and heavy-grain genotypes was provided in Tab. 6. Other types of aneuploid seedlings were rarely observed. Similar observations have been reported in Triticale (Akgun and Altindal, 2010; Tosun et al., 2003). Euploid seedlings means among light grains of all Tritipyrum-derived genotypes (parental Tritipyrum lines and $\mathrm{F}_{2}$ progenies) were significantly lower than Triticale. Euploid seedlings means among light grains of four $\mathrm{F}_{2}$ progenies were significantly $(p<0.05)$ lower than their parental genotypes. Chromosome number investigation of the light-grain class showed that Tritipyrum-derived genotypes were significantly more mitotically instable than Triticale and four $\mathrm{F}_{2}$ progenies were significantly more mitotically instable than their parental Tritipyrum genotypes.

Euploid seedlings means among heavy grains of all Tritipyrum-derived genotypes (parental Tritipyrum genotypes and $\mathrm{F}_{2}$ progenies) were significantly lower than Triticale and bread wheat as control. Euploid seedlings means among heavy grains of $\mathrm{F}_{2}$ progenies showed no significant difference with their parental Tritipyrum genotypes. These data showed that grain weight positively affected chromosome stability in segregation generations (Akgun and Altindal, 2010; Oudjehih and Boukaboub, 2000; Suarez and Favret, 1986; Tosun et al., 2003; Tsuchiya, 1973).

Fertility, 1000 grains weight and grain yield of Tritipyrum-derived genotypes have shown a significant negative correlation (Tab. 7) with mitotic instability (Elneskog Staam and Marker, 2002). Tritipyrum-derived genotypes' fertility had a significant positive correlation with their 1000-grains weight and grain yield (Kamyab et al., 2009; Shahsavand Hassani et al., 2000).
Chromosome counts of 42-chromosome hexaploid Tritipyrum-derived genotypes exhibited a considerable amount (12-32.1\%) of instability in the form of aneuploidy. The results were in agreement with Shahsavand and Hassani et al. (2000); (1997), Siahsar et al. (2011), and King et al. (1997). It has been suggested that the screening of larger and heavier grains could give a higher frequency of euploids and grain weight significantly affected mitotic chromosome stability in segregation generations. Similar investigations in other crops displayed a close relationship between grain characteristics and aneuploid frequency (Akgun and Altindal, 2010; Oudjehih and Boukaboub, 2000; Suarez and Favret, 1986; Tosun et al., 2003; Tsuchiya, 1973).

\section{Conclusions}

$F_{1}$ genotypes from 6x primary Tritipyrum/6x bread wheat showed acceptable yield components under drought stress and non-drought stress conditions compared with 6x primary Tritipyrum lines (Tab. 1 and 2). Also mitiotic stability of $F_{2}$ heavy grains was significantly similar to $6 x$ primary Tritipyrum lines (Tab. 5). Leaf water and osmotic potential and drought susceptibility index indicated better drought resistance of $F_{1}$ genotypes than that of their bread wheat parent 'Navid' cultivar. Data showed that hybridization between $6 x$ primary Tritipyrum lines and bread wheat creates unique and novel, promising generations for wheat drought resistance selection and breeding. This study indicated that the yield of primary Tritipyrum lines was equal to that of Iranian bread wheat cultivar 'Navid' and promising Triticale 4118 line under limited water conditions. In general primary Tritipyrum lines may complement the role of bread wheat in arid and semi-arid regions (Kamyab et al., 2009; Shahsavand Hassaniet al., 2006) and is a good genetic resource for wheat breeding (Allahdoo $\mathrm{et} \mathrm{al.,}$ 2009; Chen Q, 2005; Shahsavand Hassani et al., 2000); but more breeding research is needed to overcome its mitotic instability by producing the secondary Tritipyrum genotypes via crossing the primary lines with bread wheat cultivar such as 'Navid'.

\section{Acknowledgments}

The authors should give special thanks to Dr. Hossein Shahsevand Hassani for providing the various 6xTritipyrum seeds germplasm from John Innes Centre UK. 
176

\section{References}

Akgun I, Altindal N (2010). Relationship among Aneuploidy, Germination rate and seed shriveling in 6X-Triticales. Turkish J Field Crops 15(1):25-28.

Allahdoo M, Siahsar BA, Hassani HS, Pour AK, Nazhad NM (2009). The identification of $E^{b}$ chromosome in Tritipyrum Primary lines using random and semi random primers. Trakia J Sci 4:1-6.

Amiri Fahliani M, Assad MT (2005). Evaluation of Three Physiological Traits for Selecting Drought Resistant Wheat Genotypes. J Agric Sci Technol 7:81-87.

Assad MT (1997). Agricultural field experiments design and analysis. Shiraz University Press, 543 p.

Barlow EWR, Lee JW, Munns R, Smart MG (1980). Water Relations of Developing Wheat Grain. Aust J Plant Physiol 7:519-525.

Blum A (1989). Osmotic Adjustment and Growth of Barley Genotypes under Drought Stress. Crop Sci 29:230-233.

Blum A (2005). Drought resistance, water-use efficiency, and yield potential-are they compatible, dissonant, or mutually exclusive? Aus J Agric Res 56:1159-1168.

Byrne PF, Bolanos J, Edmeades GO, Eaton DL (1995). Gains from selection under drought versus multi location testing in related tropical maize populations. Crop Sci 35:63-69.

Chen Q (2005). Detection of alien chromatin introgression from Thinopyrum into Wheat using $S$ genomic DNA as a probe-A landmark approach for Thinopyrum genomic research. Cytogenetic Genome Res 109:350-359.

Elneskog Staam P, Marker A (2002). Chromosome composition, stability and fertility of Alloploid between Triticum turgidum var. carthlicum and Thinopyrum junceiforme. Hereditas 136:59-65.

Fischer RA, Maurer R (1978). Drought Resistance in Spring Wheat Cultivars 1: Grain Yield Responses. Aust J Agr Res 29:897-912.

Guinta FR, Motzo R, Deidda M (1993). Effect of drought on yield and yield components of durum wheat and Triticale in a Mediterranian environment. Field Crop Res 33:399-409.

Kamyab M, Hassani HS, Tohidinejad E (2009). Agronomic behavior of a new cereal (Tritipyrum: $\mathrm{AABBE}^{\mathrm{b}} \mathrm{E}^{\mathrm{b}}$ ) compared with modern Triticale and Iranian bread wheat cultivars. Plant Ecophysiol (2):69-80.

Keim DL, Kronstad WE(1981). Drought response of winter wheat cultivars grown under field stress conditions. Crop Sci 21:11-15.

King IP, Law CN, Cant KA, Orford SE, Reader SM, Miller TE (1997). Tritipyrum, a potential new salt-tolerant cereal. Plant Breed 116:127-132.

Kramer PJ and Boyer JS (1995). Water relations of plant and soils. Ed. Academic Press, New York.

Oudjehih B, Boukaboub A (2000). Cytogenetic study of Triticale. Agricultures 9:519-523.

Oudjehih B, Bentouati A (2006). Chromosome numbers of the 59 species of Eucalyptus L'Herit. (Myrtaceae). Caryologia
59(3):207-212.

Moustafa MA, Boersma L, Kronstad WE (1996). Response of four Spring Wheat Cultivars to Drought Stress. Crop Sci 36:982-986.

Musick JT, Jones OR, Stewart BA, Dusek DA (1994). Wateryield relationships for irrigated and dryland wheat in the U.S. Southern plains. J Agron 86:980-986.

Rajaram S, Van Ginkle M (2001). A History of Wheat Breeding, p. 579-604. In: Bonjean AP, Angus WJ (Eds). World Wheat Book. Lavoisier Publishing, Paris, France.

Shahsavand Hassani H, Caligari PDS, Miller TE (1997). Production of secondary Tritipyrum: Assessment of chromosome constitution by in situ hybridization. Proc. $3^{\text {rd. }}$ Inter Symp "New genetical approaches to crop improvement". Tando Jam, Pakistan.

Shahsavand Hassani H (1998). Development and cytogenetic studies of a potential new salt tolerant cereal, Tritipyrum. Ph.D. Thesis, The University of Reading, UK.

Shahsavand Hassani H, King IP, Reader SM, Caligari PDS, Miller TE (1998). An assessment of Tritipyrum, a new potential cereal with salt tolerance. $8^{\text {th }}$. Inter. Wheat genet. Symp, Saskatoon, Canada.

Shahsavand Hassani H, King IP, Reader SM, Caligari PDS, Miller TE (2000). Can Tritipyrum, a new salt tolerant potential amphiploid, be a successful cereal like Triticale? J Agric SciT echnol 2:177-195.

Shahsavand Hassani H, Reader SM, Miller TE (2006). Agronomical and adoption characters of Tritipyrum lines in comparison with Triticale and Iranian wheat. Asian J Plant Sci 5(3):553-558.

Sharma SN, Sain RS, Sharma RK (2003). The genetic control of the flag leaf length in normal and late sown durum wheat. J Agric Sci 141:323-331.

Siahsar BA, Maryam A, Hassani HS (2011). Genetic variation among and within Tritipyrum (Thinopyrumbessarabicum $\times$ Triticum durum) lines using PCR-based molecular markers. Cien Inv Agr 38(1):127-135.

Suarez EY, Favret EA (1986). Aneuploidy as an explanation of high values of phenotypic variability in commercial wheat varieties. Cereal Res Comm 14(3):229-235.

Talebi R, Fayaz F, Naji AM (2009). Effective selection criteria for assessing drought stress tolerance in durum wheat (Triticum durum Desf.). Gen Appl Plant Physiol 35:64-74.

Tosun M (1999). Karyotype analysis in hexaploid Triticale. Turkish J Agric For 23:943-949.

Tosun M, Halilo-Lu K, Pinar SM, Sasöz S (2003). Test weight, kernel shriveling, and aneuploidy frequency in Triticale. New Zealand J Agric Res 46:27-30.

Tsuchiya T (1973). Frequency of euploids in different seed size classes of hexaploid Triticale. Euphytica 22(3):592 -599.

Zadoks JC, Chang TT, Konzak CF (1974). A decimal code for the growth stages of cereals. Weed Res 14:415-421.

Zhong-hu H, Rajaram S (1994). Differential responses of bread wheat characters to high temperature. Euphytica 72:197203. 